\title{
A Cost-Effective Atomic Force Microscope for Undergraduate Control Laboratories
}

\author{
Colin N. Jones and Jorge Gonçalves, Member, IEEE
}

\begin{abstract}
This paper presents a simple, cost-effective and robust atomic force microscope (AFM), which has been purposely designed and built for use as a teaching aid in undergraduate controls labs. The guiding design principle is to have all components be open and visible to the students, so the inner functioning of the microscope has been made clear to see. All of the parts but one are off the shelf, and assembly time is generally less than two days, which makes the microscope a robust instrument that is readily handled by the students with little chance of damage. While the scanning resolution is nowhere near that of a commercial instrument, it is more than sufficient to take interesting scans of micrometer-scale objects. A survey of students after their having used the AFM resulted in a generally good response, with $80 \%$ agreeing that they had a positive learning experience.
\end{abstract}

Index Terms-Atomic force microscope (AFM), control, microscopy, system identification, teaching laboratory.

\section{INTRODUCTION}

$\mathbf{T}$ RADITIONAL control experiments, such as the inverted pendulum and the three-tank system, are useful pedagogical tools for illustrating basic control concepts. However, modern control theory is used in diverse fields ranging from nanotechnology to large-scale fermentation, and the authors believe that bringing examples of applications from these and other exciting areas will help to draw future generations to control engineering. Such an example is the atomic force microscope (AFM), which can produce topological surface scans with subnanometer resolutions and is used in areas ranging from materials science to biology. This paper introduces a new and simple AFM suitable for control experiments. A lab for senior undergraduates is also outlined, in which students go through a complete design cycle of system identification and controller design and implementation for the piezo-positioning stage of the microscope before proceeding to take various scans.

The world's first AFM was invented in 1985 [1], and since then, they have found applications in many fields, among them surface science, nanotechnology, polymer science, semiconductor materials processing, microbiology, and cellular biology.

Manuscript received March 28, 2008; revised April 02, 2009. First published September 09, 2009; current version published May 05, 2010.

C. N. Jones is with the Department of Electrical Engineering, Swiss Federal Institute of Technology Zürich, 8092 Zürich, Switzerland (e-mail: cjones@ee. ethz.ch).

J. Gonçalves is with the Department of Engineering, University of Cambridge, Cambridge CB2 1PZ, U.K. (e-mail: jmg77@ @am.ac.uk).

Color versions of one or more of the figures in this paper are available online at http://ieeexplore.ieee.org.

Digital Object Identifier 10.1109/TE.2009.2021390
Accurate commercial AFMs are generally very expensive and require a high level of understanding to use effectively. These two factors mean that it is not generally feasible to use commercial instruments in an undergraduate setting. Furthermore, these devices are fully enclosed and factory-tuned, which reduces their instructive value since they would have to be used as a "black-box" sensor.

For these reasons, a new tool has been developed to introduce undergraduate engineering students to atomic force microscopy, specifically to the relevant control aspects involved. Three design principles were adhered to:

- The device must be open, meaning that every aspect of the system must be both visible and adjustable by the students. Furthermore, it must be sufficiently robust to withstand such inexpert adjustments.

- The equipment itself must be inexpensive.

- The device must be able to be assembled and maintained by a technician without in-depth knowledge of atomic force microscopy.

As can be seen from Fig. 1, the microscope is indeed very open and all parts are visible. Furthermore, all but one simple part are off the shelf, and this remaining part can be acquired from the authors at no cost or constructed in a few minutes on a computer numerical controlled (CNC) machine. The total cost is approximately $£ 7,200$, as outlined in Table I. This price could be reduced through substitution of a less expensive piezo-positioning stage, although the effect of such a substitution on the accuracy of the AFM would have to be determined from the manufacturer's specifications. (While the authors have no direct experience with different piezo stages, experience with the device suggests that up to a 50\% reduction in accuracy would still produce a tool useful for an undergraduate laboratory.) The extremely simple nature of the microscope means that it can be assembled and calibrated from scratch in one or two days by someone with no special knowledge of electronics or microscopy. A detailed parts list and assembly instructions are available from the authors.

The design of this device was heavily influenced by the microscope developed in the Massachusetts Institute of Technology (MIT) Biological Engineering Department (Cambridge, MA) [2], and the authors are thankful for the assistance provided by M. Shusteff and S. Manalis. While the MIT device addresses the first two of the three design principles, it differs from that proposed here in two important aspects. First, the AFM proposed in this paper uses all off-the-shelf components save for one, while that in [2] requires several more custom-built parts and electronics. The commercial components in the proposed device lead to a fast build time and easy maintenance 


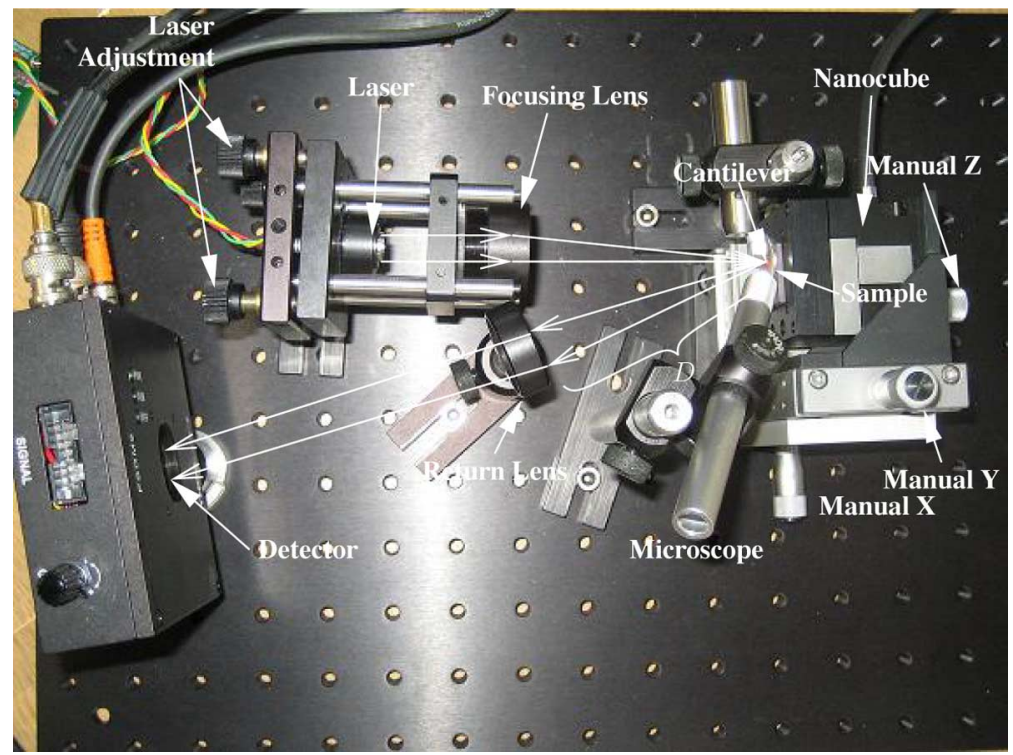

Fig. 1. Photograph of the AFM.

TABLE I

APPROXIMATE COST OF MAJOR COMPONENTS

\begin{tabular}{lr} 
PC with NI-DAQ system & $£ 1,500$ \\
Optical components & $£ 700$ \\
Piezo-positioning stage and associated & $£ 4,500$ \\
electronics & $£ 500$ \\
Laser position detector & $£ 7,200 \quad(\approx 15,000$ USD $)$ \\
\hline Total &
\end{tabular}

without an increase in cost. Second, the AFM developed at MIT is designed around a specialized interdigitated cantilever, which must be custom-made at one of the few fabrication labs available to academics, such as the one at MIT. Because such a lab was not available to the authors, or indeed to most control academics, the AFM was designed to use standard, inexpensive, and reliable commercial probes. ${ }^{1}$

The remainder of the paper is split into two sections. Section II provides an overview of the microscope and its basic functions. Section III covers the lab that is currently running at the University of Cambridge, Cambridge, U.K., using the developed device, in which students go through an entire controller design procedure from modeling to implementation and finally to using their controllers to take scans of various materials.

\section{ATOMIC ForCE MicRoscope}

\section{A. Principles of Atomic Force Microscopy}

An AFM functions by dragging a sharp pin, mounted on a beam (the cantilever), back and forth over a sample. As it moves, the contours of the sample cause the cantilever to bend up and down, and by measuring the height of the cantilever, the surface features of the sample can be determined. The

${ }^{1}$ The authors of [2] provide fabrication instructions and indicate on their Web site [3] that the National Nanotechnology Infrastructure Network (NNIN) may be able to provide microfab facilities for academic use. They also offer interested educators small quantities of the interdigitated probes at a nominal cost.
AFM works because this height can be measured with extreme accuracy - down to a resolution of a few picometers on a commercial AFM. This resolution is achieved by bouncing a laser off the tip of the cantilever, which is a polished mirror. The angle of the reflected laser changes as the cantilever bends, and the position of the reflected laser spot on a detector is proportional to the height of the sample and inversely proportional to the length of the cantilever. This inverse proportionality gives the AFM its resolution, since the cantilever is produced using photolithography, and is generally only a few microns long.

The class of instruments called AFMs contain many variations on this theme. In particular, the tip of the cantilever is generally never in contact with the surface of the sample, but instead is held close enough that it is effected by the strong atomic force of the sample (hence the name). This lab uses the crudest approach, in which the tip is in direct contact with the surface, and as a result, the resolution is significantly lower than that which can be achieved on a commercial AFM. However, even this crude instrument is capable of measuring features on the submicron scale.

The geometry of a simple AFM is shown in Fig. 2; note that the scale is off by several orders of magnitude. As the height of the sample changes and the cantilever moves upward by a distance $h$, the laser's angle of reflection $\theta$ changes and the laser moves along the detector by a distance $r$. Some simple computations show that the position $r$ of the laser on the detector as a function of the change in height $h$ of the cantilever is

$$
r=\frac{2 l(D+d \cos (\theta)) h+2 d \sin (\theta) h^{2}}{\cos (\delta) l^{2}-\cos (\delta) h^{2}+2 \sin (\delta) l h}
$$

where $D$ is the distance between the cantilever and the detector and $\delta$ is the misalignment angle between the detector and the incoming laser. Note that the height $h$ is much smaller than the length of the cantilever $l$ (nanometers versus microns), and the distance $D$ between the detector and the cantilever is several orders of magnitude larger than the length of the cantilever $l$. 


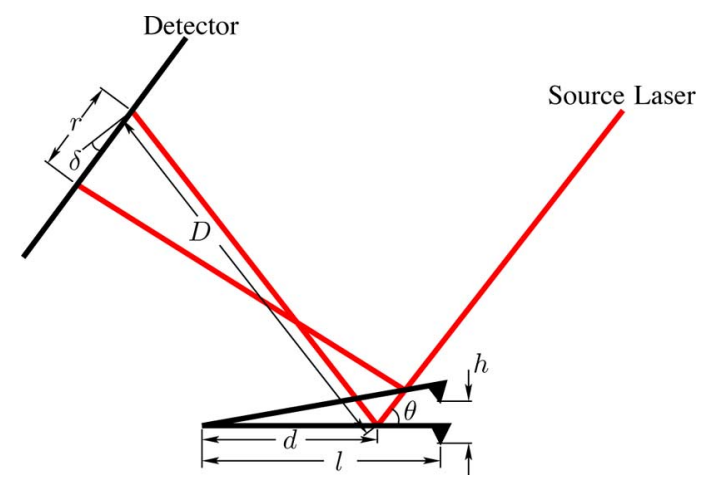

Fig. 2. Geometry of an optical lever. (Figure not to scale.)

As a result, one can accurately approximate (1) with the linear relation

$$
r \approx \frac{2 D}{l} h \approx 2000 \cdot h
$$

which is approximately a gain of 2000 times for this instrument ( $D \approx 10 \mathrm{~cm}, l \approx 100 \mu \mathrm{m}$ ). It is this optical gain that gives the microscope its impressive magnification since a change in the height of the sample of $100 \mathrm{~nm}$ results in the laser moving $200 \mu \mathrm{m}$, which is easily detectable.

\section{B. Laboratory AFM}

The AFM developed for this lab is shown in Fig. 1. As can be seen from the photograph, the basic design principle throughout the development was to make all parts as large, open, and visible to the students as possible. This approach is the opposite to that taken in commercial microscopes since smaller, enclosed devices are more resistant to vibration and, as a result, take better scans. A significant amount of accuracy has therefore been sacrificed in this interest of providing clarity for the students. The path from the laser through to the cantilever and back to the detector is marked on the photograph and matches that shown in Fig. 2.

The physical parts of the microscope labeled on Fig. 1 are as follows.

- Laser: This Is a simple 635-nm, 5-mW diode laser, the same type that is in many laser pointers.

- Laser Adjustment: These manual knobs allow the angle of the laser to be changed very accurately. They are used to target the laser on the tip of the cantilever.

- Focusing Lens: This lens can be manually rotated to focus the laser to as tight a spot as possible. While a commercial AFM will focus extremely accurately on the back of the cantilever, it has been found that the very rough targeting allowed by this manual approach is more than accurate enough for a teaching device.

- Cantilever: The cantilever is mounted on a semicircular disk that is held in place with a small magnet, which is an industry standard approach. The cantilever itself is too small to be seen with the naked eye, but can be viewed through the attached microscope. The cantilever used is a standard probe purchased from Veeco Instruments Inc. for approximately $£ 20$ per probe. These do break during the lab, at a rate of about one probe per month per group of two students.

- Piezo Positioning: The "Nanocube" is a piezoelectric positioning system that allows the sample to be moved back and forth underneath the cantilever with a (nominal) 1-nm accuracy. This is an off-the-shelf system that includes a $3^{\circ}$-of-freedom piezo-positioning and measurement module, along with the appropriate amplification and signal conditioning unit.

- Sample: The sample sits uncovered on a microscope slide, which is glued to a metal washer. The washer is attached to the surface of the Nanocube magnetically (a fridge magnet is simply glued to the surface of the cube).

- Manual X,Y,Z: These knobs allow coarse movement of the sample up to $2 \mathrm{~cm}$. They are used to pull the sample back from the cantilever in order to change the slide or to adjust the gross location of the sample being scanned.

- Microscope: The microscope is a very inexpensive fixed $\times 50$ magnification. The focus is adjusted by simply pushing the microscope closer or farther away, and then locked in place with a hand-tightened screw. While there is very little chance that a student could point the laser down the microscope due to the geometry, it is conceivable that with sufficient misguided determination, one could achieve this and so potentially damage their eyes. To prevent this, a small filter is placed on the microscope to avoid any possibility of eye damage.

- Return Lens: The light coming off the cantilever is in a cone shape. The return lens is symmetric to the focusing lens and recollimates the light into a beam.

- Detector: The linear detector measures the position of the laser spot as it moves continuously across its 10 -mm detection range. Note that a standard AFM uses a fixed sensor location and continuously adjusts the height of the cantilever in order to ensure that the position of the laser stays fixed. It was found that such an approach is very sensitive to adjustment errors and that keeping the sample fixed and purchasing a slightly more expensive sensor that can measure linear position made the microscope much more robust against alignment errors.

As one can see from Fig. 1, virtually all components used are standard and can be found in most stores that sell general optics equipment.

There are two key differences between the design of this microscope and a standard commercial one. First, the entire system has been turned on its side. The sample is held vertically, and the laser travels in a horizontal path rather than a vertical one. This makes it a lot easier to position all of the components without the need of mounting everything on a custom-made vertical panel. The downside is that the sample is no longer held in place by gravity - a complication that is easily overcome with magnets, although this does prevent the scanning of wet samples.

The second, more important difference is that the cantilever is held in a fixed position while the sample moves. In a standard AFM, the sample moves in the plane of the sample, and the cantilever itself is moved in the direction perpendicular to it. This arrangement is adopted for several reasons. First, in most modern AFMs, the cantilever is actually vibrating at a very high 


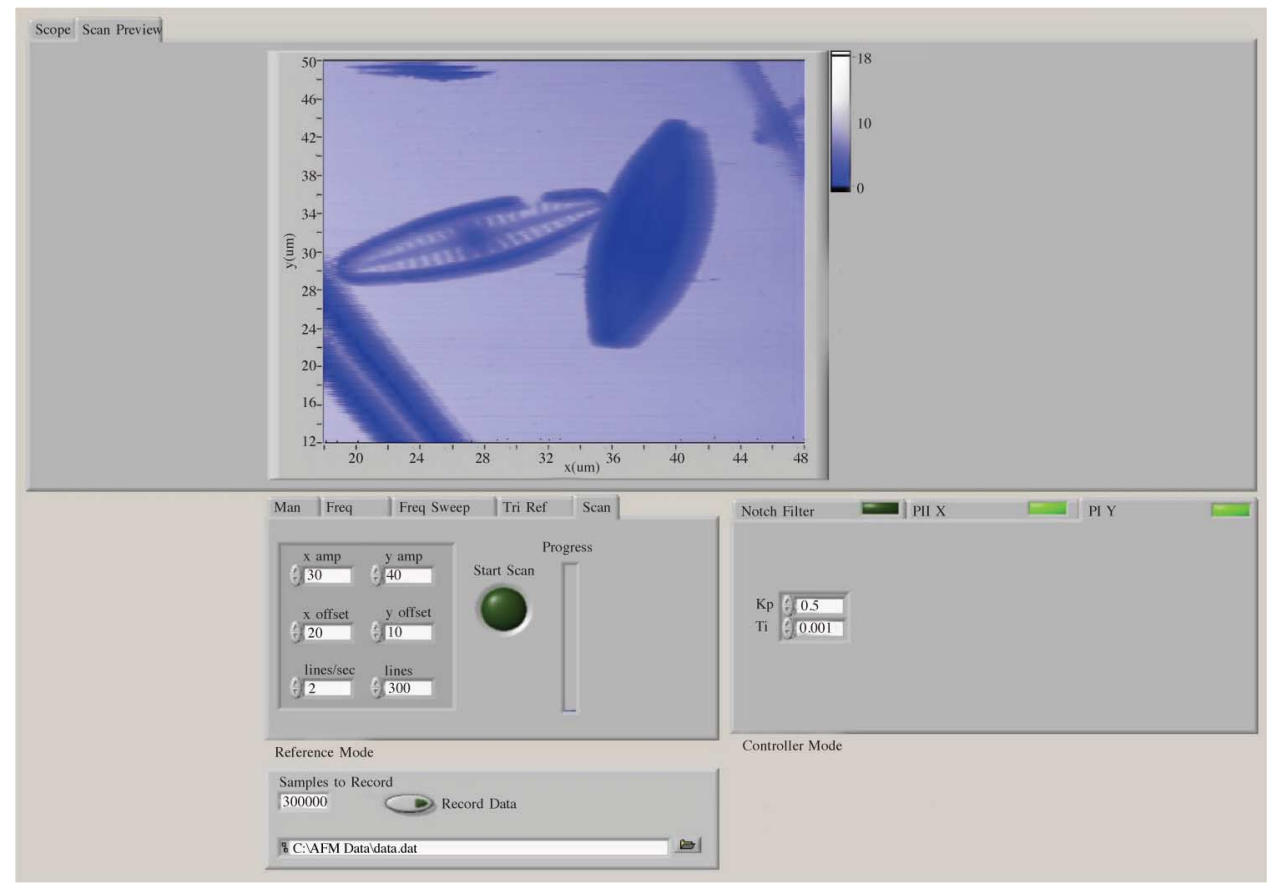

Fig. 3. Screenshot of AFM control interface.

frequency, and the system detects changes in this frequency as the cantilever is brought near to the sample, rather than the direct contact that is used here. Second, the distance of the cantilever above the sample is kept constant by moving it up and down in response to changes in the height of the sample. Both of these actions require that the vertical position of the cantilever be actuated by a piezo system. For the design described here, it was felt that such a system would both add complexity to the design and, more importantly, block the student's view. As a result, in the proposed design, the cantilever is held in a fixed location, and as can be seen from Fig. 1, this allows the students a very clear view of the workings of the system. Although it is possible to adjust the distance between the sample and the cantilever during a scan by moving the sample on its piezo stack, it was decided not to do this as it adds complexity to the project. The result is that the system cannot scan samples with large height variations since the cantilever would bend too much. Given that the detector can measure the position of the laser in a 10-mm window, a simple calculation using (2) tells us that the maximum change in height allowed for the sample is $10 \mathrm{~mm} / 2000=5 \mu \mathrm{m}$, which is not restrictive.

The only component that needs to be custom-built is the magnetic holder for the cantilever. This is a simple item to produce and can be made in about an hour on a CNC machine. Full drawings or a prebuilt component are available from the authors.

\section{Electronics}

The electronics are kept extremely simple by using existing commercial components as much as possible, in order to reduce construction time and maintenance effort. The main component is the three-axis piezo-positioning system, marked "Nanocube"

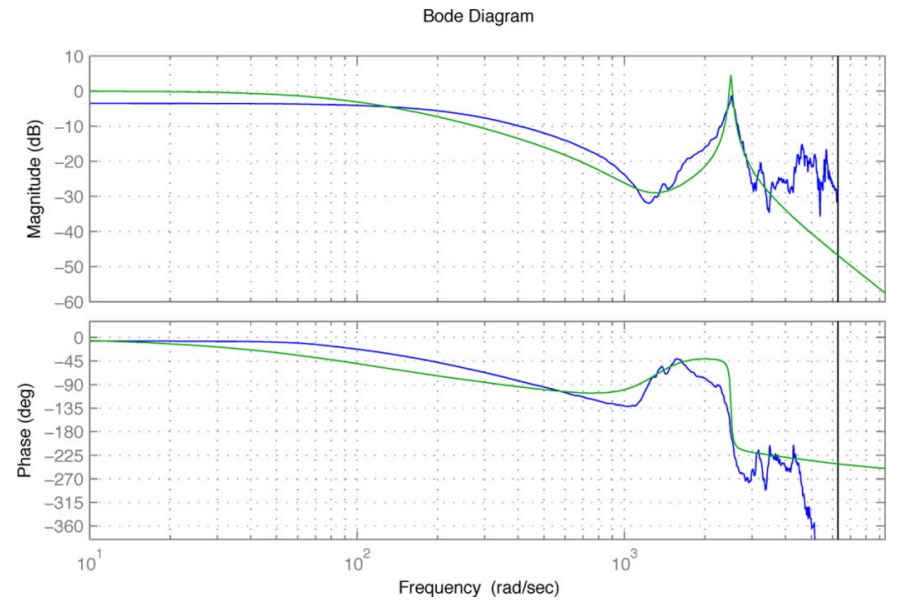

Fig. 4. Measured spectral model (jagged line) and LTI system (smoother line).

in Fig. 1. The Nanocube offered by PI technologies ${ }^{2}$ has proven to be reliable, robust, and trivial to interface to a National Instruments data acquisition board. The cube is a compact device that can position the sample in a $100 \mu \mathrm{m} \times 100 \mu \mathrm{m} \times 100 \mu \mathrm{m}$ range with an accuracy of $1 \mathrm{~nm}$. The system comes with an amplifier and signal conditioning system that reduces the construction effort to nothing more than attaching the cables and a few bolts.

\section{Software}

The AFM is controlled through a custom-made software tool running in LabView. The main interface can be seen in Fig. 3, which allows the students to view the various signals coming from the AFM as an oscilloscope or as a basic preview of the image while taking a scan. A software signal generator is provided, which can create the various types of signals needed in

\footnotetext{
${ }^{2}$ See "Nanocube" at www.physikinstrumente.com (last viewed May 4, 2009).
} 


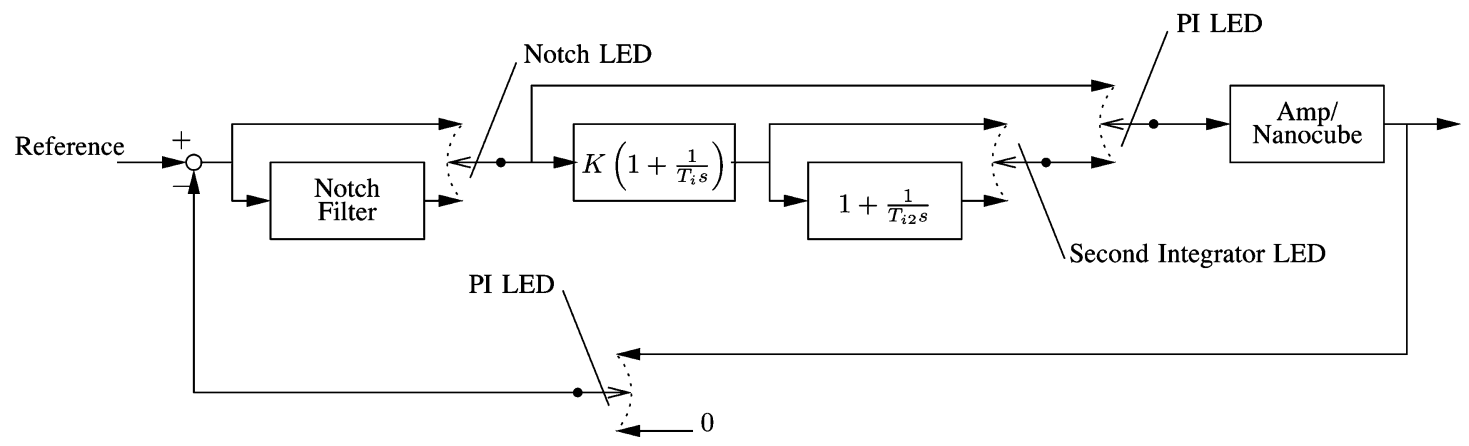

Fig. 5. Controller structure of the AFM scope program.
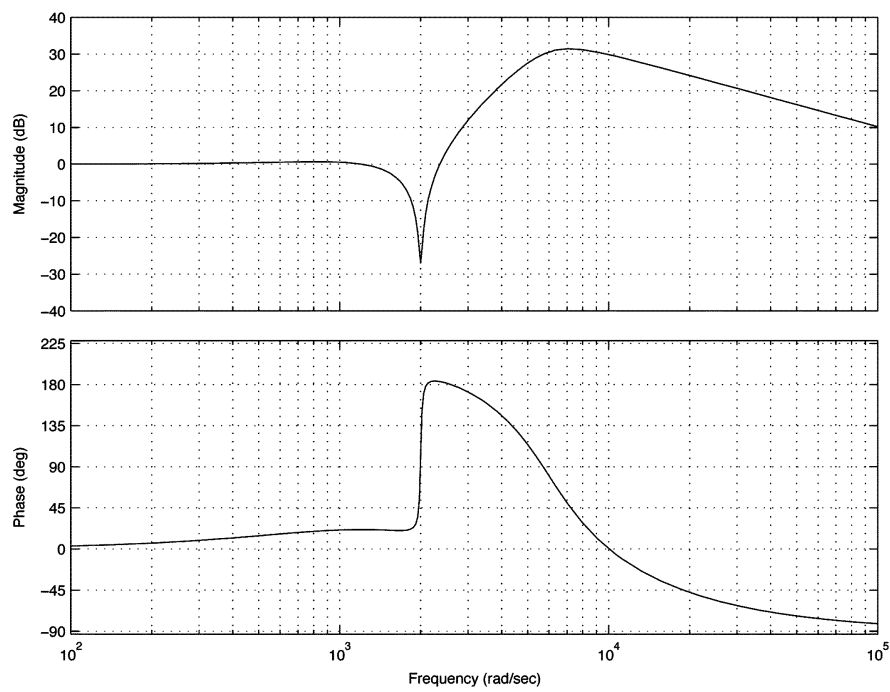

Fig. 6. Bode plot of notch filter.

the lab: square, triangle and sinusoidal waves, frequency sweep, and raster-scan reference sequences. The PI controllers for the piezo-positioning system can be switched on and off and tuned, and the parameters of more general digital filters can be entered. Finally, at any point, the students can choose to record a sequence of samples to the disk for later analysis in Matlab.

The controller is a soft real-time system running in LabView under Windows. The lack of a dedicated processor, or hard realtime guarantees, means that it is possible for there to be jitter in the control signal. However, without taking any special precautions, it was found that LabView is more than capable of running the controller and data collection at a rate of $2 \mathrm{kHz}$ on a standard Pentium IV machine without any noticeable jitter, even when several compute-intensive programs such as Matlab are running.

Once data has been collected, there are several post-processing, analysis, and design activities that the students must do in the Matlab environment. A small Matlab toolbox has been provided for this purpose, the functions of which are outlined where appropriate in the following sections.

The LabView software and Matlab toolbox are freely available in open-source form from the authors.
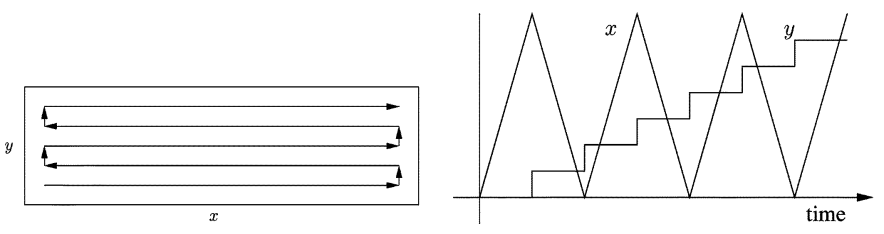

Fig. 7. The scanning path of the $x$ - and $y$-axis.

\section{LABORATORY EXPERIMENTS}

Various experiments can be carried out using the AFM in the areas of signals and systems, control and modeling, etc. This section will briefly outline the modeling and control laboratory created and currently running at the University of Cambridge. It should be emphasized, however, that the instrument does not produce scans to a quality or repeatability that it could be used for any experiments whose primary target is microscopy, apart from demonstrating the basic concepts.

The main objective of the lab is for the students to go through a complete design procedure for a lightly damped mechanical system. By this stage, the students will have taken one linear control theory course and will be studying a second. The purpose of the lab is to reinforce the course knowledge by providing the practical experience of an entire design cycle consisting of a system identification and modeling phase, controller design, and finally implementation.

The lab takes the students four weeks to complete and consists of four supervised and 16 unsupervised $h$ per week. There are four AFM setups, with two students to each device. Each pair takes ownership of a single device for the entire four-week period, which removes the requirement for the students to recalibrate the device each week.

\section{A. Gain Measurement and Calibration}

In the first section of the lab, the students familiarize themselves with the working principles of the AFM through reading and examination of the equipment and learn the basic operation and calibration of the system. They derive the gain equation (1) from first-principles, then use various techniques to measure and compute the gain of their AFM ranging from using a ruler to a calibrated strain gauge. 


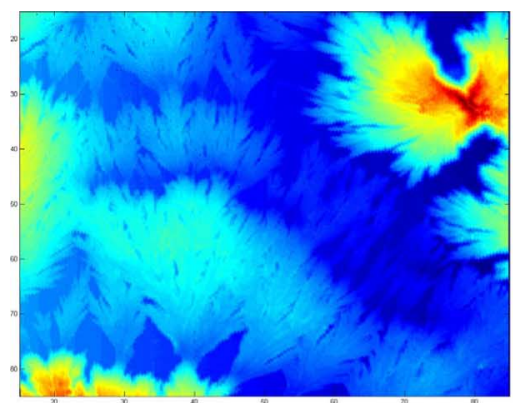

(a)

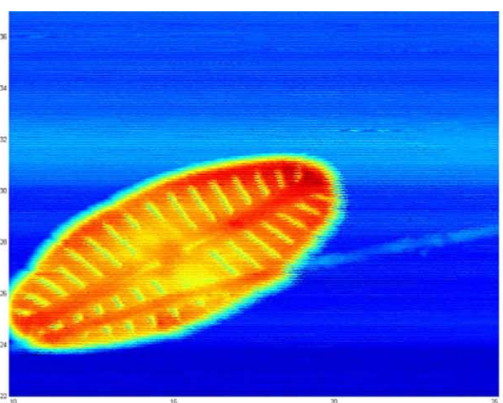

(b)

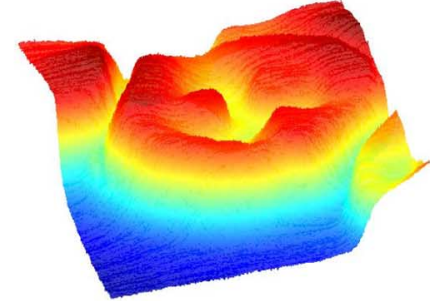

(c)

Fig. 8. Sample scans. (a) Calcium crystals $(70 \mu \mathrm{m} \times 70 \mu \mathrm{m})$. (b) Diatom $(15 \mu \mathrm{m} \times 16 \mu \mathrm{m})$. (c) Author's dried blood cell ( $8 \mu \mathrm{m} \times 8 \mu \mathrm{m})$.

TABLE II

STUDENT QUESTIONNAIRE

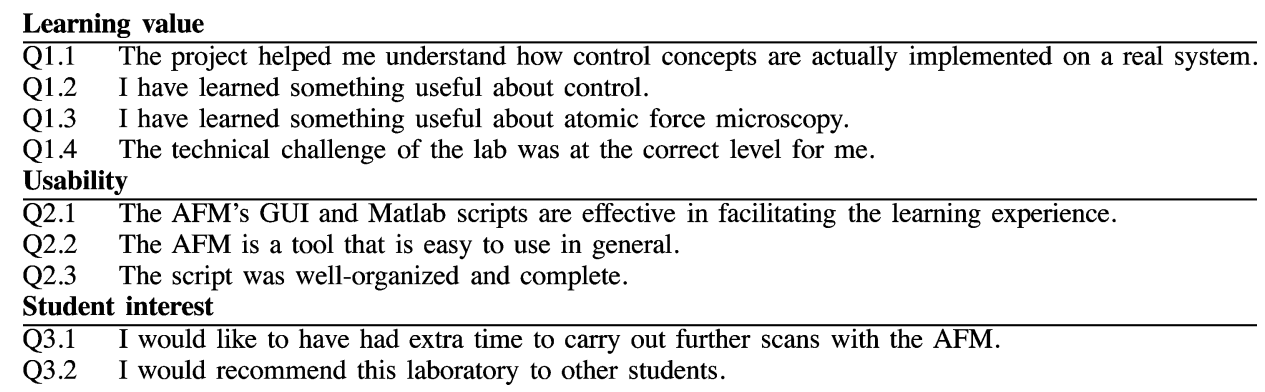

TABLE III

Student Questionnaire Results in Percentage of Total Number OF ANSWERS PER SECTION

\begin{tabular}{l|rrrrr} 
& $\begin{array}{r}\text { Strongly } \\
\text { Disagree }\end{array}$ & Disagree & Neutral & Agree & $\begin{array}{c}\text { Strongly } \\
\text { Agree }\end{array}$ \\
\hline Learning value & 0 & 0 & 7 & 38 & 55 \\
Usability & 0 & 5 & 12 & 26 & 57 \\
Student interest & 0 & 0 & 11 & 29 & 61
\end{tabular}

\section{B. Modeling}

The main portion of the developed lab examines the control of a piezo element, which is challenging due to its having a strong resonance peak. In this section, the students go through various methods of system identification in order to determine a reasonable model, the target being to identify the gain and bandwidth of the system as well as the primary resonance frequencies. In the next section, the developed models are used to design suitable controllers.

Most first-year control courses focus on teaching frequency and Bode techniques, and so the modeling section of the lab investigates various methods for developing useful frequency models from noisy data. The students begin by estimating system response rates from step tests and locating resonance peaks by driving the system with sinusoidal inputs. Once they have a rough idea of the system response, they collect data resulting from a frequency sweep and build a spectral model from first principles in Matlab. With a filtered spectral model in hand, they can then determine an appropriate structure for a parametric linear time invariant (LTI) model and manually fit the parameters.

The Bode plots of the measured spectral and LTI models are shown in Fig. 4. One can see that there is a significant resonance at $\approx 2.2 \mathrm{krad} / \mathrm{s}$. In the commercial amplifier purchased with the piezo stage, there is actually a low-pass filter on the input that prevents this resonance from being excited. In order for the students to observe the resonance and compensate for it, this filter was detuned in the amplifier. ${ }^{3}$ Due to this detuning and subsequent controller design, the final result of the project is a controller that has a larger bandwidth and better disturbance rejection properties than the original commercial controller.

\section{Controller Design}

The controller design breaks into two sections. First, a compensator for the resonance peak seen in the previous section is designed, and then two PI controllers are tuned for the $x$ - and $y$-scanning directions (in the plane of the sample). The layout of the control system is shown in Fig. 5.

The resonance seen in the previous section is a common problem that arises in many mechanical systems, so it is of benefit to the students to see a practical solution. The main issue with the resonance peak is that the gain of the system is greater than one when the phase falls below $180^{\circ}$, which causes instability when the loop is closed. A common solution to this problem is to add a notch filter to the system, which has a response as shown in Fig. 6. The idea is not to place the notch on top of the resonance peak, as the student's intuition would suggest, but rather to place it just before the peak so that the phase advance provided by the filter pushes the open loop phase above $180^{\circ}$ at the crossover frequency. This results in a controller that actively damps disturbances at the resonance frequency, rather than one that simply prevents the controller

\footnotetext{
${ }^{3}$ This detuning was achieved by opening the amplifier case and turning the potentiometer controlling the filter, as detailed in the product specifications.
} 
from injecting energy at the resonance frequency (which is, in fact, what the commercial amplifier was doing).

The second phase of the controller design consists of tuning two PI controllers for the two different axes of the AFM. Two controllers are required because the different axes track different input signals while performing a raster scan across the sample, as shown in Fig. 7. From the figure, it can be seen that the ' $x$-axis controller must track step inputs, and the $y$-axis ramps. From their first control course, the students know from the internal model principle that the controller must contain one integrator for the step and two for the ramp. A pragmatic approach is taken in this phase of the lab, in which the students first determine reasonable ranges for the gains on their PI and PII controllers in Matlab, before implementing and manually fine-tuning them on the AFM until they produce satisfactory responses with fast rise-times and small overshoot.

\section{Scanning}

There are two sections to the scanning portion of the lab. In the first, the students use their controllers to run scans on various prepared samples, ranging from diatoms (single-celled organisms with hard shells) and calcium crystals to blood and hair. Inevitably, the more enthusiastic students will also scan everything within reach and then bring in new, stranger samples the next day.

The second portion of the scanning task takes them back into Matlab to do post-processing on the images. These tasks include estimating the plane of the sample and rotating it to level (there is always some misalignment in all AFMs), resampling the measured data onto a regular grid and special, and temporal filtering to remove noise.

A few sample scans are shown in Fig. 8. These demonstrate that the AFM is more than capable of imaging microscopic scales to a quality that will interest students, although a commercial AFM would be able to resolve at least two or three orders of magnitude smaller again.

\section{Assessment AND EVAluATION}

The pedagogical value of the discussed laboratory, the usability of the equipment, and the interest of the students in the experiment were evaluated through a survey of the students taking the course in 2008 . There are only 16 students who take the lab each year, and so the number of respondents is relatively low (14). The survey does, however, give an indication of the benefit to senior engineering undergraduates.

The questions asked under each heading are given in Table II, and the results summarized by section are shown in Table III. Over $80 \%$ of the students agreed with the statements made in each section of the questionnaire, indicating a positive overall reaction to the laboratory. Undue emphasis should not, however, be placed on this initial survey, due to the small sample size.

\section{CONCLUSION}

This paper has presented a simple atomic force microscope, which is used to teach undergraduate engineering students basic control concepts. The microscope has a very open and robust design, allowing students to see and adjust every aspect without concern for damage. It has been used from the year 2007 at the University of Cambridge to train third-year students, who have found it an interesting and educational experiment.

\section{ACKNOWLEDGMENT}

The authors would like to thank National Instruments for providing the data acquisition hardware and the Labview software and the Cambridge-MIT Institute for funding the development of the microscope.

\section{REFERENCES}

[1] G. Binnig, C. Quate, and C. Gerber, "Atomic force microscope," Phys. Rev. Lett., vol. 56, no. 9, pp. 930-933, 1986.

[2] M. Shusteff, T. Burg, and S. Manalis, "Measuring boltzmann's constant with a low-cost atomic force microscope: An undergraduate experiment," Amer. J. Phys., vol. 74, no. 10, pp. 873-879, 2006.

[3] M. Shusteff and S. Manalis, "An atomic force microscope (AFM) for the instructional laboratory," Last viewed May 4, 2009 [Online]. Available: http://www.media.mit.edu/nanoscale/courses/AFMsite/index.html

Colin N. Jones received the Bachleur and Master of Applied Science degrees at the University of British Columbia, Vancouver, Canada, in 2001, and the Ph.D. degree from the University of Cambridge, Cambridge, U.K., in 2005.

In 2006, he was a Post-Doctoral Researcher in the Automatic Control Laboratory at the ETH, Zurich, Switzerland, and has been a Senior Researcher since 2008. He founded the company Apex Optimization, focusing on scheduling and optimization of human resources in 2007. His current research interests are in the areas of high-speed predictive control, computational geometry, energy management, and optimal scheduling.

Jorge Gonçalves (M'07) received the Licenciatura (five-year S.B.) degree from the University of Porto, Porto, Portugal, in 1993, and the M.S. and Ph.D. degrees from the Massachusetts Institute of Technology (MIT), Cambridge, in 1995 and 2000, respectively, all in electrical engineering and computer science.

He held two Post-Doctoral positions, first at MIT for seven months, then at the Control and Dynamical Systems Division, California Institute of Technology, Pasadena, from May 2001 to March 2004. Since April 2004, he has been a Lecturer in the Information Engineering Division, Department of Engineering, University of Cambridge, Cambridge, U.K. Since 2005, he has also been a Fellow of Pembroke College, Cambridge, U.K. His research interests include modeling, analysis, and control of complex and hybrid systems-in particular, modeling and analysis in systems and synthetic biology, collaborating with biologists in different areas such as circadian rhythms and gene regulatory networks.

Dr. Gonçalves was the recipient of the Best Student Paper Award at the Automatic Control Conference, Chicago, IL, June 2000. 